\title{
CORRIGENDUM \\ Prophylactic HPV vaccination: past, present, and future - CORRIGENDUM
}

\section{P. E. CASTLE AND M. MAZA}

doi:http://dx.doi.org/10.1017/S0950268815002198. Published online: 2 October 2015.

In Table 1 of the above-mentioned article [1] the units for 'Vaccine composition' (column 1, line 5) are incorrect. They should be $\mu \mathrm{g}$, rather than $\mathrm{mg}$.

In Figure 1, the orange bar for 'ATP all cervical precancer' should indicate 42.7\%. In the caption for Figure 1, reference [29] should be [27]. The correct version of Figure 1 and caption are given below.

The authors apologize for these errors.

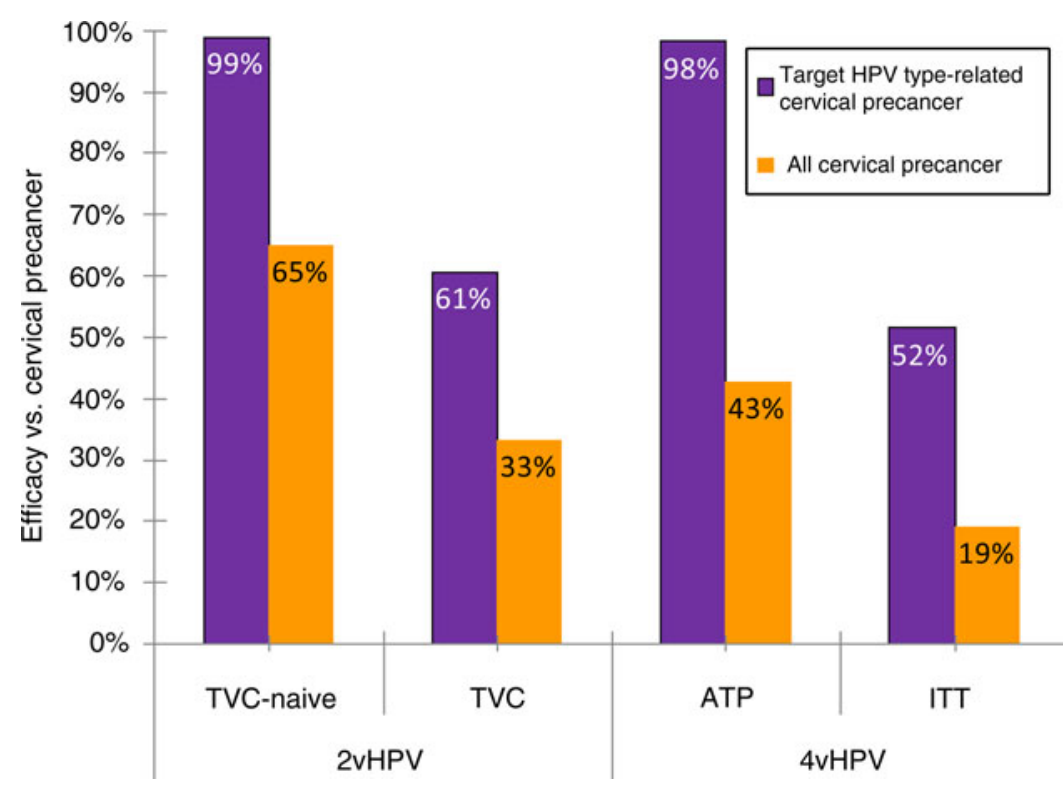

Fig. 1. A comparison of vaccine efficacy against targeted HPV type-related (purple bars) and all cervical precancer (CIN2, CIN3, or AIS) (orange bars) by 2vHPV [27], and 4vHPV [28], for the HPV-naive populations (TVC-naive and ATP, respectively) and the entire vaccinated cohort (TVC and ITT, respectively). TVC, Total vaccine cohort; ATP, attention to protocol; ITT, intention to treat.

\section{REFERENCE}

1. Castle PE, Maza M. Prophylactic HPV vaccination: past, present, and future. Epidemiology and Infection 2016; 144: 449-468. doi:10.1017/S0950268815002198. Published online: 2 October 2015. 